\title{
Reconstruction from Planar Motion Image Sequences with Applications for Autonomous Vehicles
}

\author{
H. Stewénius, M. Oskarsson, and K. Åström \\ Centre For Mathematical Sciences, \\ Lund University, Lund,Sweden \\ \{stewe, magnuso, kalle\}@maths.lth.se
}

\begin{abstract}
Vision is useful for the autonomous navigation of vehicles. In this paper the case of a vehicle equipped with multiple cameras with non-overlapping views is considered. The geometry and algebra of such a moving platform of cameras are considered. In particular we formulate and solve structure and motion problems for a few novel cases. There are two interesting minimal cases; three points in two platform positions and two points in three platform positions. We also investigate initial solutions for the case when image lines are used as features. In the paper is also discussed how classical algorithms such as intersection, resection and bundle adjustment can be extended to this new situation. The theory has been tested on synthetic and real data with promising results.
\end{abstract}

\section{Introduction}

One example of successful use of computer vision for autonomous vehicle navigation is that of laser guided navigation. Here the sensor can be seen as that of a one-dimensional retina, which has all-around sight in a plane, (typically) a horizontal plane. During the last two decades many geometrical problems and system design problems have been investigated and solved for this system. Algorithms using a pre-calculated map and good initial position was demonstrated in [8]. In 1991 a semi-automatic method for map making was constructed [1] and in 1996 a fully automatic system for map making was made, 2]. Theory for structure and motion recovery is detailed in [3].

During the last decade there has also been many attempts at making fully automatic structure and motion systems for ordinary camera systems. A good overview of the techniques available for structure and motion recovery can be found in [7]. Much is known about minimal cases, feature detection, tracking and structure and motion recovery. Many automatic systems rely on small image motions in order to solve the correspondence problem, i.e. association of features across views. In combination with most cameras' small fields of view, this limits the way the camera can be moved in order to make good $3 \mathrm{D}$ reconstruction. The problem is significantly more stable with a large field of view $[9$. This has spurred research in so called omnidirectional cameras. 
In this paper we investigate an alternative approach to vision-based structure and motion system. We consider a vehicle equipped with multiple cameras. This gives a large combined field of view with simple and cheap cameras. Another advantage is that there are no moving parts contrary to laser scanner sensors. If the cameras can be placed so that the focal points coincide, then the geometrical problems are identical to that of a single camera. Also if the cameras are positioned so that they have a large field of view in common, i.e. a stereo setup, there are known techniques for calculating structure and motion. In this paper we consider cameras where neither of these constraints have to be satisfied.

Consider cameras that are fixed to a vehicle and consider images taken by these cameras at different times, where the vehicle has moved. Assume that a number of corresponding points (possibly in different images) are measured. Then there are a number of problems that are interesting to look at.

1. Calibration. Assume that each camera sees enough points to calculate its own relative camera motion. Since the relative motion of all cameras is the same, how many cameras and views are needed to solve for the common motion. When is it possible to solve for the cameras' relative positions?

2. Structure and motion. Assume that the cameras' positions relative to the vehicle are known, but not the vehicle's motion, represented by a transformation matrix $\mathbf{T}_{i}$. Given a number of corresponding points, how should one calculate the world points $\mathbf{U}_{j}$ and the transformations $\mathbf{T}_{i}$ from image data?

3. Intersection. Assume that the cameras' positions relative to the vehicle are known, and that also the vehicle's motion is known. Assume that a number of corresponding points are measured, how should one calculate the world points $\mathbf{U}_{j}$ from image data?

4. Resection. Assume that the cameras' positions relative to the vehicle are known, but not the vehicle's motion, represented by a transformation matrix $\mathbf{T}_{i}$. Assume that a number of corresponding points (possibly in different images) are measured, how should one calculate the transformations $\mathbf{T}_{i}$ from image data and known world points $\mathbf{U}_{j}$ ?

In this paper we assume that the calibration of the cameras relative the vehicle has been done. In the experiments in section 6 the calibration was done manually. One could also consider autocalibration approaches similar to the approach in [5], where the problem of aligning video sequences was adressed, or the calibration problems in robotics [13] which are similar in nature to the calibration of cameras relative a vehicle.

Multiple camera platforms have been studied in [10], where a discrete constraint linking two camera motions and image points, similar to the fundamental constraint for two views of a configuration of points, 7 was formulated. However, there is no analysis of that constraint. Similarly in 4 multi-camera motions are considered but the analysis is concentrated to that of motion flow. In this paper we study and solve some of the minimal cases for multi-camera platforms. Such solutions are of paramount importance as initial estimates to bootstrap automatic structure and motion recovery systems. 


\section{Geometry of Multi-camera Platforms}

The standard pinhole camera model is used,

$$
\lambda \mathbf{u}=\mathbf{P} \mathbf{U}
$$

where the camera matrix $\mathbf{P}$ is a $3 \times 4$ matrix. A scene point $\mathbf{U}$ is in $\mathcal{P}^{3}$ and a measured image point $\mathbf{u}$ is in $\mathcal{P}^{2}$. As the platform moves the cameras move together. This is modeled as a transformation $\mathbf{T}_{i}$ between the first position and position $i$. In the original coordinate system the camera matrix for camera $k$ at position $i$ is $\mathbf{P}_{k} \mathbf{T}_{i}$.

It is assumed here that the camera views do not necessarily have common points. In other words, a point is typically seen by only one camera. On the other hand it can be assumed that in a couple of neighboring frames a point can be seen in the same camera. Assume here that point $j$ is visible in camera $k$. The measurement equation for the $n$ points at $m$ positions is then

$$
\lambda_{i j} \mathbf{u}_{i j}=\mathbf{P}_{k} \mathbf{T}_{i} \mathbf{U}_{j}, \quad j=1, \ldots, n, i=1, \ldots, m .
$$

Problem 1. Given $n$ image points from $m$ different platform positions $\mathbf{u}_{i j}$, and the camera matrices $\mathbf{P}_{k}$ the structure and motion problem is to find reconstructed points, $\mathbf{U}_{j}$, and platform transformations, $\mathbf{T}_{i}$ :

$$
\mathbf{U}_{j}=\left[\begin{array}{c}
X_{j} \\
Y_{j} \\
Z_{j} \\
1
\end{array}\right] \text { and } \mathbf{T}_{i}=\left[\begin{array}{cccc}
a_{i} & b_{i} & 0 & c_{i} \\
-b_{i} & a_{i} & 0 & d_{i} \\
0 & 0 & 1 & 0 \\
0 & 0 & 0 & 1
\end{array}\right] \text { with } a_{i}^{2}+b_{i}^{2}=1
$$

such that $\lambda_{i j} \mathbf{u}_{i j}=\mathbf{P}_{k} \mathbf{T}_{i} \mathbf{U}_{j}, \quad \forall i=1, \ldots, m, j=1, \ldots, n$ for some $\lambda_{i j}$.

The special form of $\mathbf{T}_{i}$ is due to the planar motion.

Counting the number of unknowns and constraints for generalized cameras restricted to planar motion we have the following, given $n 3 \mathrm{D}$-points and $m$

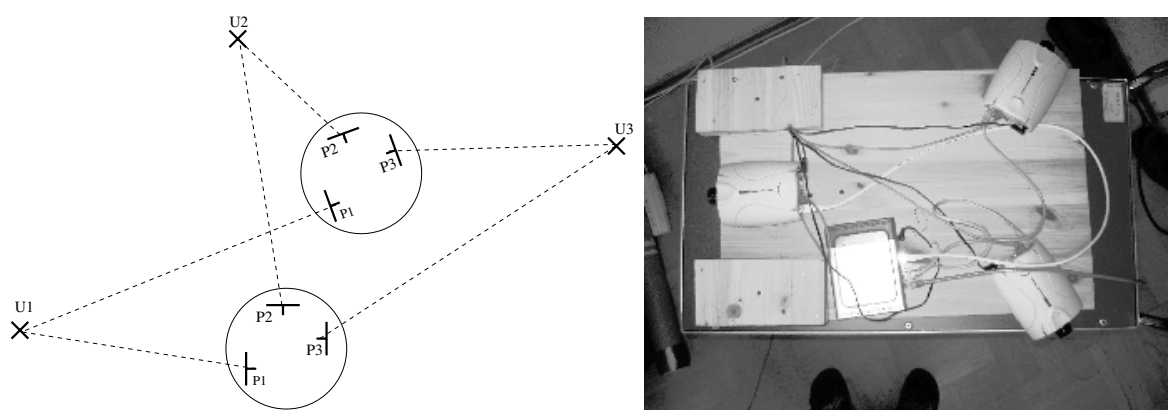

Fig. 1. Three calibrated cameras with constant and known relative positions taking images of three points at two platform positions 
cameras. Each point has three degerees of freedom and each camera has three degrees of freedom. Each point in each camera gives two constraints. In addition we have the freedom to specify a Euclidean coordinate system. We have fixed a plane, which leaves three degrees of freedom. So in order to solve a system,

$$
2 m n \geq 3 m+3 n-3 .
$$

There are two interesting cases, where equality holds,

Theorem 1. For two sets of three rays each that are allowed to move planarly there are in general three or one real solution to problem 1 .

Theorem 2. For three sets of two rays each that are allowed to move planarly there are in general three or one real solution to problem 1 .

The two cases will be discussed in sections 2.1 and 2.2 respectively.

\subsection{Solving for Two Sets of Three Rays}

All observations are assumed to be given as Plücker vectors $\left(q, q^{\prime}\right)$, see e.g. 11 . Assuming that the first camera is at the origin, that is, $\left(a_{0}, b_{0}, c_{0}, d_{0}\right)=(1,0,0,0)$ there are now only four motion variables left $\left(a_{1}, b_{1}, c_{1}, d_{1}\right)$. For convenience we will not use the indices on these variables.

Given a motion $\mathbf{T}$ and Plücker coordinates for the observations these can be inserted into the generalized epipolar constraint 10

$$
q_{1}^{T} R q_{2}^{\prime T}+q_{1}^{T} R[t]_{\times} q_{2}+q_{1}^{\prime T} R q_{2}=0
$$

where $[t]_{\times}$is the cross-product matrix formed from $t$ such that $[t]_{\times} v=t \times v$.

Inserting our three observed point pairs in equation (5) gives 3 equations each of degree 2 . We also have that $a^{2}+b^{2}=1$. This gives a total of 4 equations in 4 unknowns.

The three first equations are in the monomials $(a c, a d, b c, b d, a, b, c, d, 1)$. Using linear elimination on these equations gives a way to express $d$ as a linear combination of $(a, b, c, 1)$ and $d$ can thus be eliminated without increasing the degree. This leads to two equations in $a, b$ and $c$ named $f_{1}$ and $f_{2}$. A third polynomial comes from the rotation constraint, $f_{3}=a^{2}+b^{2}-1$.

The nine polynomials $f_{1}, f_{2}, f_{3}, a f_{1}, a f_{2}, b f_{1}, b f_{2}, b f_{3}, c f_{3}$ can be represented as

$$
M\left[a^{2} b, a^{2} c, a b^{2}, a b c, a^{2}, a b, a c, b^{2}, b c, b^{3}, b^{2} c, a, b, c, 1\right]^{T}
$$

where $M$ is a $9 \times 15$ matrix. Performing a Gauss-Jordan elimination on $M$ gives a GrevLex Gröbner basis. For details on Gröbner bases and their use in elimination theory see e.g. [6].

Given the Gröbner basis the action matrix $m_{a}$ for multiplication with $a$ can be extracted. The matrix $m_{a}$ is $4 \times 4$ and the left eigenvectors give the solutions to $(a, b, c, 1)$. One of the solutions corresponds to not moving the vehicle, which leaves three solution of which two may be complex. The remaining motion variable $d$ is computed using back-substitution. 


\subsection{Solving for Three Sets of Two Rays}

For each time $i=1,2,3$ and point $j=1,2$ we have

$$
\pi_{i j} \mathbf{T}_{i} \mathbf{U}_{j}=0 \text { and } \pi_{i j}^{\prime} \mathbf{T}_{i} \mathbf{U}_{j}=0,
$$

where $\pi_{i j}$ and $\pi_{i j}^{\prime}$ are planes in the camera coordinate system defining the observed ray, calculated from the image point, $\mathbf{T}_{i}$ the position of the vehicle and $\mathbf{U}_{j}$ the scene point. The coordinate system is fixed by

$$
\mathbf{U}_{1}=\left[\begin{array}{llll}
0 & 0 & z_{1} & 1
\end{array}\right]^{T}, \mathbf{U}_{2}=\left[\begin{array}{llll}
0 & y_{2} & z_{2} & 1
\end{array}\right]^{T} .
$$

We also know that

$$
a_{i}^{2}+b_{i}^{2}=1, i=1,2,3 .
$$

Equation (7) can now be written

$$
\left[A+B\left(\left\{\left(a_{i}, b_{i}\right)\right\}\right)\right] X=0 \text { where } X=\left[z_{1}, z_{2}, c_{1}, d_{1}, c_{2}, d_{2}, c_{3}, d_{3}, 1, y_{2}\right]^{T}
$$

and $A$ is a matrix with coefficients computed from the observations. The vector $B\left(\left\{\left(a_{i}, b_{i}\right)\right\}\right)$ is linear in $\left\{\left(a_{i}, b_{i}\right)\right\}$ and has coefficients computed from the observations. For this $12 \times 10$ matrix to have non-trivial solutions all $10 \times 10$ sub-determinants must be zero. All these sub-determinants are linear and homogeneous in $\left\{\left(a_{i}, b_{i}\right)\right\}$. The system can now be written as

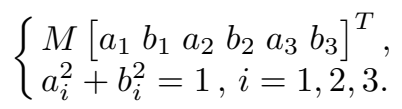

The matrix $M$ is linear in $\left(a_{1}, a_{2}, a_{3}, b_{1}, b_{2}, b_{3}\right)$ and has rank 3 . We can thus compute $\left(a_{1}, a_{2}, a_{3}\right)$ as a linear function of $\left(b_{1}, b_{2}, b_{3}\right)$. This is inserted into equation (9), giving three equations of order two in $\left(b_{1}, b_{2}, b_{3}\right)$,

$$
Q\left[b_{1}{ }^{2}, b_{2}{ }^{2}, b_{3}{ }^{2}, b_{1} b_{2}, b_{1} b_{3}, b_{2} b_{3}, 1\right]^{T}=0
$$

where $Q$ is a $3 \times 7$ matrix. In order to compute the solutions we compute the multiples of these three polynomials by $1, x^{2}, x y, x z, y^{2}, y z, z^{2}$ and arrange the coefficients into a matrix. By performing Gauss-Jordan elimination on this matrix we get the elements of the Gröbner basis needed for computing the action matrix for multiplication by $b_{3}{ }^{2}$ on polynomials containing only monomials of even order. Solving the eigenvalue problem gives the three solutions to $\left(b_{3}{ }^{2}, b_{2} b_{3}, b_{1} b_{3}\right)$. From this the values of $b_{3}$ are computed and by division, $b_{1}$ and $b_{2}$ as well.

When the $\left\{b_{i}\right\}$ have been computed, the $\left\{a_{i}\right\}$ can be computed using backsubstitution in equation (11) and the remaining unknowns can then be computed using back-substitution in equation (10).

\subsection{Three Positions and Lines}

When viewing a line $\mathbf{l}$ in an image the corresponding scene line is constrained to lie on a scene plane $\pi=\mathbf{P}^{T} \mathbf{l}$. Two positions give no constraints on the platform 
motion, but with three positions there is a constraint that three planes intersect in a line. This constraint can be written

$$
\operatorname{rank}\left[\begin{array}{lll}
\mathbf{T}_{1}^{T} \pi_{1} & \mathbf{T}_{2}^{T} \pi_{2} & \mathbf{T}_{3}^{T} \pi_{3}
\end{array}\right]=2 .
$$

This implies that all four sub-determinants of size $3 \times 3$ are zero. The subdeterminant that involves rows 1, 2 and 3 can be interpreted as the constraint that three lines (the intersection with the planes and the plane at infinity) intersect in a point (the direction of the space line). This constraint does not involve translation components of $\mathbf{T}$, since the plane at infinity is unaffected by translation. Introduce the following parametrisation for the three transformation matrices,

$$
\mathbf{T}_{1}=\left[\begin{array}{llll}
1 & 0 & 0 & 0 \\
0 & 1 & 0 & 0 \\
0 & 0 & 1 & 0 \\
0 & 0 & 0 & 1
\end{array}\right], \mathbf{T}_{2}=\left[\begin{array}{ccccc}
a_{2} & e_{2}-b_{2} & 0 & c_{2} \\
b_{2}-e_{2} & a_{2} & 0 & d_{2} \\
0 & 0 & e_{2} & 0 \\
0 & 0 & 0 & e_{2}
\end{array}\right], \mathbf{T}_{3}=\left[\begin{array}{cccc}
a_{3} & e_{3}-b_{3} & 0 & c_{3} \\
b_{3}-e_{3} & a_{3} & 0 & d_{3} \\
0 & 0 & e_{3} & 0 \\
0 & 0 & 0 & e_{3}
\end{array}\right]
$$

Here we have used homogenized versions of the transformations matrices $\mathbf{T}_{2}$ and $\mathbf{T}_{3}$. There is a constraint that the first $2 \times 2$ block is a rotation matrix, i.e.

$$
a^{2}+(b-e)^{2}-e^{2}=a^{2}+b^{2}-2 b e=0 .
$$

By dehomogenizing using $b=1$, we see that $e=\left(1+a^{2}\right) / 2$. Using

$$
b_{2}=1, b_{3}=1, e_{2}=\left(1+a_{2}^{2}\right) / 2, e_{3}=\left(1+a_{3}^{2}\right) / 2,
$$

the plane at infinity constraint is of the form

$$
\left(k_{22} a_{2}^{2}+k_{12} a_{2}+k_{02}\right) a_{3}^{2}+\left(k_{21} a_{2}^{2}+k_{11} a_{2}+k_{01}\right) a_{3}+\left(k_{20} a_{2}^{2}+k_{10} a_{2}+k_{00}\right)=0 .
$$

Thus there are two unknowns on the rotation parameters $a_{2}$ and $a_{3}$. Using two different lines, two constraints are obtained. The resultant of these two polynomials with respect to $a_{3}$ is an eight degree polynomial. Two of the roots to this polynomial are $\pm i$. The remaining six roots can be complex or real. For each solution on $a_{2}$, the variable $a_{3}$ can be obtained from equation (16). Then $\left(b_{2}, b_{3}, e_{2}, e_{3}\right)$ follow from (15).

Once the rotation is known it can be corrected for. The rank constraint (13) is then linear in the translation parameters $\left(c_{2}, c_{3}, d_{2}, d_{3}\right)$. Using four lines it is then possible to obtain these motion parameters uniquely in general.

Observe that by counting equations and unknowns, three lines would be a minimal case. However, since the constraint at infinity only involves the rotation parameters, these are found already with two lines. The third line gives one additional constraint on the rotation parameters, which then becomes overdetermined, and a third constraint on the four translation parameters. The fourth line gives yet one additional constraint on the rotation parameters and the fourth constraint on the four translation parameters.

There are a couple of interesting degenerate cases. If the camera centers lie in the same horizontal plane, then lines in that plane give no constraints. Vertical 
lines give no constraints on rotation. A platform viewing a set of vertical lines is equivalent to a platform motion with 1D retina cameras. For this situation the minimal case is six lines in three views. There are in general 39 solutions to this situation.

\section{$3 \quad$ Intersection}

Generalization of the intersection algorithm [7] to this new situation is straightforward. When both calibration $\mathbf{P}_{1}, \ldots, \mathbf{P}_{K}$ and platform motion $\mathbf{T}_{1}, \ldots, \mathbf{T}_{m}$ is known it is straightforward to calculate scene points coordinates $\mathbf{U}$ from image measurements by intersecting view-lines. A linear initial solutions is obtained by solving

$$
\left[\begin{array}{c}
\mathbf{u}_{1} \times \mathbf{P}_{k} \mathbf{T}_{1} \\
\ldots \\
\mathbf{u}_{n} \times \mathbf{P}_{k} \mathbf{T}_{n}
\end{array}\right] \mathbf{U}=0
$$

in a least squares sense. This solution can then be refined by non-linear optimization, cf. section 5 .

\section{Resection}

Generalization of the resection algorithm [7] is slightly more complex since the view lines do not go through a common points (the focal point) as in the ordinary camera resection algorithm.

Here we introduce a direct method for finding an initial estimate to the resection problem based on a linearized reprojection error. The idea is to solve

$$
\mathbf{u}_{j} \times \mathbf{P}_{k} \mathbf{T U}_{j}=0, \quad j=1, \ldots, n,
$$

which is linear in $\mathbf{T}$. In our case there are non-linear constraints on $\mathbf{T}$, so we use the theory for constrained optimization to find the optimal solution under the constraints.

Parameterize the platform motion as in (3), using parameters $x=(a, b, c, d)$. The constraints $\mathbf{u}_{j} \times \mathbf{P}_{k} \mathbf{T} \mathbf{U}_{j}=0$ is linear in these parameters. The linear constraints can be rewritten $f=M x=0$. However there is a non-linear constraint $g=a^{2}+b^{2}-1=0$. Initial solution to the resection problem can be found by solving

$$
\min _{x g=0} \sum_{j}\left|\mathbf{u}_{j} \times \mathbf{P}_{k} \mathbf{T}(x) \mathbf{U}_{j}\right|^{2} .
$$

Introduce the Lagrangian $L(x, \lambda)=|M x|^{2}+\lambda g$. The solution to (19) can be found by $\nabla L=0$. Here

$$
\nabla_{x} L=\left[\begin{array}{ll}
A & B \\
C & D
\end{array}\right]\left[\begin{array}{l}
a \\
b \\
c \\
d
\end{array}\right]+\lambda\left[\begin{array}{c}
2 a \\
2 b \\
0 \\
0
\end{array}\right]=0 .
$$


Here one may solve for $(c, d)$ as

$$
\left[\begin{array}{l}
c \\
d
\end{array}\right]=-D^{-1} C\left[\begin{array}{l}
a \\
b
\end{array}\right] .
$$

Inserting this in the above equation gives

$$
\left(A-B D^{-1} C+2 \lambda I\right)\left[\begin{array}{l}
a \\
b
\end{array}\right]=0 .
$$

Here there is a non-trivial solution to $(a, b)$ if and only if $-2 \lambda$ is one of the two eigenvalues of $A-B D^{-1} C$. For these two solutions $(a, b)$ is determined up to scale. The scale can be fixed by $a^{2}+b^{2}=1$. Finally $(c, d)$ can be found from (21). Of the two solutions, only one is a true solution to (19). This gives a reasonably good initial estimate on the resection parameters. This estimate is then improved by minimizing reprojection errors. Experience shows that only a few iterations are needed here.

\section{Bundle Adjustment}

Note that the discussion in the previous sections has focused on finding initial estimates of structure and motion. In practice it is necessary to refine these estimates using non-linear optimization or bundle adjustment, cf. [12, 7]. The generalization of bundle adjustment to platform motions is straightforward. One wants to optimize platform motions $\mathbf{T}_{i}$ and scene points $\mathbf{U}_{j}$ so that reprojection error is minimized. The fact that the platform has a very large field of view makes bundle adjustment much better conditioned than what is common.

\section{Experimental Verification}

Experiments on both simulated and real data were conducted.

A virtual room was was constructed as a cube and points randomly placed on the surfaces of the cube. The synthetic vehicle was moved along the $z$-plane taking "pictures" of this room.

The experiment was carried out at different levels of noise added to the image point measurements. The first experiment was to add errors of magnitude up to $1 / 100$ for a calibrated camera, that is with a known field of view of 0.6 and assuming 300 pixels image width corresponding to an error of 5 pixels. The result is shown in figure 2, with the true room to the left, and the reconstruction to the right.

In an experiment with real data a system with three digital video cameras was assembled and moved along a corridor with markers on the walls. Tracking was done by following these markers and some additional manual tracking. 

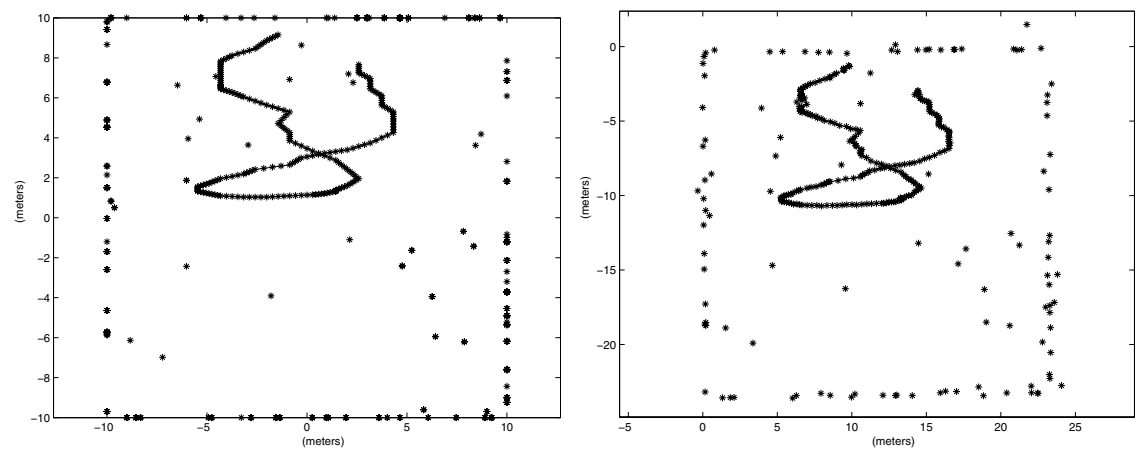

Fig. 2. True room to the left path compared to reconstruction on the right

A reconstruction is shown in figure 3. The structure point which we seem to be passing through is a structure point which has a very high variance, as its measured view lines are almost collinear with the locations from which it is observed. The residuals in reprojection for this reconstruction are shown in figure 3. The size of the residuals are in the order of the error in a calibrated camera.
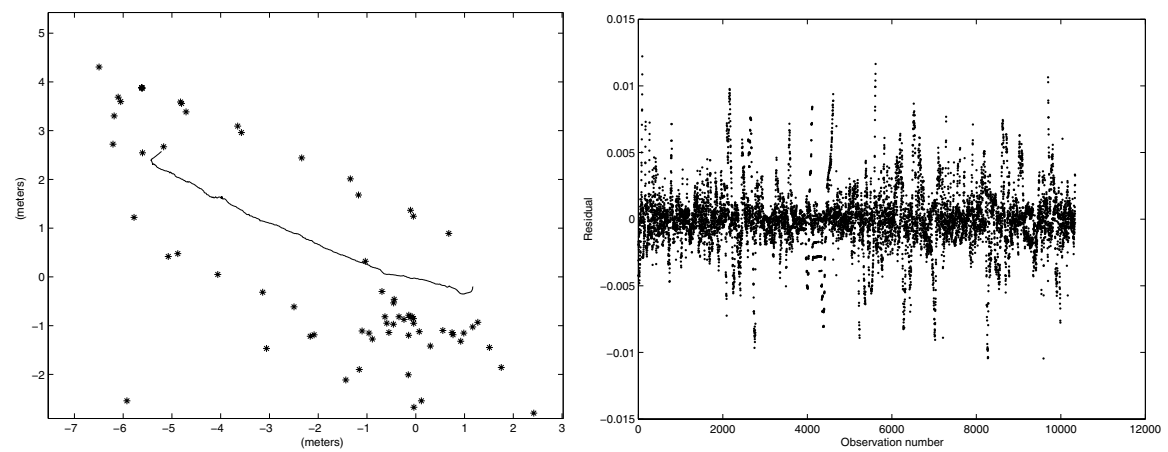

Fig. 3. Reconstruction and residuals for real data

\section{Conclusions}

In this paper we study the visual geometry of a moving platform with multiple cameras (typically pointed outwards) in general positions and orientations. For planar motion of such platforms the case of two motions and at least three points is solved, the case of three motions and two points is solved, as is the case of three motions and a number of lines.

In the experimental validation it is demonstrated how these algorithms, combined with novel algorithms for resection, intersection and bundle adjustment, are used in automatic structure and motion recovery using such platforms. The validation is done both for synthetic and real data. 
Future work includes generalizations of the above ideas to that of full (nonplanar) camera motion as well as testing and developing fully automatic systems for map-making.

\section{References}

1. K. Åström. Automatic mapmaking. In 1st IFAC, International Workshop on Intelligent Autonomous Vehicles, 1993. Selected papers.

2. K. Åström. Invariancy Methods for Points, Curves and Surfaces in Computational Vision. PhD thesis, Dept of Mathematics, Lund University, Sweden, 1996.

3. K. Åström and M. Oskarsson. Solutions and ambiguities of the structure and motion problem for 1d retinal vision. Journal of Mathematical Imaging and Vision, 12(2):121-135, 2000.

4. P. Baker, C. Fernmuller, and Y. Aloimonos. A spherical eye from multiple cameras (makes better models of the world). In Proc. Conf. Computer Vision and Pattern Recognition, Hawaii, USA, 2001.

5. Y. Caspi and M. Irani. Alignment of Non-Overlapping sequences. In Proc. 8th Int. Conf. on Computer Vision, Vancouver, Canada, pages 76-83, 2001.

6. D. Cox, J. Little, and D. O'Shea. Using Algebraic Geometry. Springer Verlag, 1998.

7. R. I. Hartley and A. Zisserman. Multiple View Geometry in Computer Vision. Cambridge University Press, 2000.

8. K. Hyyppä. Optical navigation system using passive identical beacons. In Louis O. Hertzberger and Frans C. A. Groen, editors, Intelligent Autonomous Systems, An International Conference, Amsterdam, The Netherlands, 8-11 December 1986, pages 737-741. North-Holland, 1987.

9. M. Oskarsson and K. Åström. Accurate and automatic surveying of beacon positions for a laser guided vehicle. In Proc. European Consortium for Mathematics in Industry, Gothenburg, Sweden, 1998.

10. R. Pless. Using many cameras as one. In Proc. Conf. Computer Vision and Pattern Recognition, Madison, USA, 2003.

11. J. G. Semple and G. T. Kneebone. Algebraic Projective Geometry. Clarendon Press, Oxford, 1952.

12. C. C. Slama. Manual of Photogrammetry. American Society of Photogrammetry, Falls Church, VA, 1980.

13. H. Zhuang, Z. Roth, and R. Sudhakar. Simultaneous robot/world and tool/flange calibration by solving homogeneous transformations of the form ax=yb. IEEE Trans. on Robotics and Automation, 10(4):549-554, 1994. 